\title{
Transatlantica
}

Revue d'études américaines. American Studies Journal

\section{"Lemme tell you a couple of three things" : la littérature selon Les Sopranos (1999-2007)}

\section{Bernard Genton}

\section{(2) OpenEdition}

\section{Journals}

Édition électronique

URL : https://journals.openedition.org/transatlantica/6182

DOI : $10.4000 /$ transatlantica.6182

ISSN : $1765-2766$

Éditeur

Association française d'Etudes Américaines (AFEA)

\section{Référence électronique}

Bernard Genton, " "Lemme tell you a couple of three things" : la littérature selon Les Sopranos

(1999-2007) », Transatlantica [En ligne], 2 | 2012, mis en ligne le 13 mai 2013, consulté le 06 avril 2023. URL : http://journals.openedition.org/transatlantica/6182 ; DOI : https://doi.org/10.4000/ transatlantica.6182

Ce document a été généré automatiquement le 6 avril 2023.

\section{c) (†) $९$}

Creative Commons - Attribution - Pas d'Utilisation Commerciale - Pas de Modification 4.0 International - CC BY-NC-ND 4.0

https://creativecommons.org/licenses/by-nc-nd/4.0/ 


\title{
"Lemme tell you a couple of three things" : la littérature selon Les Sopranos (1999-2007)
}

\author{
Bernard Genton
}

1 La série Les Sopranos baigne dans une atmosphère littéraire créée et entretenue par une succession de références au corpus traditionnel des œuvres enseignées dans les lycées et les premières années des universités. Il est donc intéressant d'explorer plus avant un thème qui n'a pas semblé retenir l'attention des commentateurs, à savoir celui des rapports qu'entretient et que manifeste cette série « de qualité » avec la littérature et le livre, envisagés ici comme objets culturels extérieurs au genre télévisuel, revêtus du prestige de la légitimité et de l'autorité sociale. Les Sopranos ont quelque chose à dire sur la littérature et, plus généralement, sur la culture légitime, et ce de plusieurs manières différentes. En premier lieu, et c'est le point de départ de la présente étude, la série évoque d'une manière insistante le rôle et l'importance de l'étude des œuvres littéraires dans l'éducation et dans les processus d'ascension sociale. En même temps, elle ne cesse de poser le problème de la supériorité morale, ou de la "distinction ", que confère la possession d'un capital culturel et langagier acquis. Enfin, elle s'intéresse, au moins de manière objective, à la capacité (ou à l'incapacité) de la culture livresque à faire face à la concurrence de la culture populaire audiovisuelle - sur le plan de la satisfaction du besoin de récit et de modèles d'expérience et de vie comme du point de vue de la valeur esthétique ${ }^{1}$.

2 Ces questionnements complexes et imbriqués nécessitent deux détours préalables. Dans un premier temps, nous évoquerons les origines et les conditions de la création des Sopranos ainsi que les principales caractéristiques d'une réception critique dans l'ensemble très positive, voire dithyrambique. Nous tenterons ensuite de dresser brièvement la typologie des références culturelles qui marquent la série. C'est alors que nous tenterons de répondre aux trois principales questions posées : comment la série appréhende-t-elle le rôle de la culture apprise dans les processus d'éducation et d'ascension sociale? La possession d'un capital culturel est-elle pour autant une 
garantie de prestige et, simultanément, un signe extérieur de domination morale? Enfin, nous essaierons de savoir ce que nous disent à leur manière Les Sopranos sur les rapports entre la culture légitime et la culture populaire.

\section{Les Sopranos (1999-2007)}

3 Diffusée par la chaîne câblée payante HBO de 1999 à 2007, déclinée en 6 saisons et 86 épisodes, la série Les Sopranos met en scène près de 120 personnages dans un cadre fixe et dans une temporalité légèrement décalée par rapport à l'actualité. Les lieux, l'ambiance visuelle, la nature et le rythme des dialogues restent constants, ce qui n'exclut pas quelques variations géographiques ou stylistiques: l'action se déplace parfois, en Californie, en Nouvelle-Angleterre, à Naples, à Paris, sans jamais rompre les amarres avec le New Jersey, tandis que quelques séquences oniriques complètent la tonalité réaliste de l'ensemble (Nelson). Les Sopranos sont une œuvre collective contrôlée par David Chase, producteur et scénariste de télévision reconnu et récompensé par plusieurs distinctions au moment où il se lance dans l'aventure. Né David De Cesare en 1945 dans une famille modeste de la communauté italo-américaine du New Jersey, David Chase a fait des études supérieures à l'Université de New York et à Berkeley. Sur 86 épisodes au total, le showrunner en a écrit ou co-écrit 28 et entièrement réalisé deux, le premier (The Sopranos) et le dernier (Made in America). Sur la vingtaine des autres réalisateurs qui signent les différents épisodes, les plus sollicités sont eux aussi des vétérans de la télévision et des séries nés dans les années 1940 ou 1950 dans le Nord-Est des États-Unis, et ils sont tous diplômés de l'enseignement supérieur : Tim Van Patten (une vingtaine d'épisodes), John Patterson (13 épisodes), Allen Coulter (12 épisodes), Alan Taylor (9 épisodes), Jack Bender (4 épisodes) et Daniel Attias (3 épisodes). Figurent dans cette liste quelques exceptions: Steve Buscemi, acteur et réalisateur proche des milieux du cinéma indépendant, est responsable de 4 épisodes, dont le fameux Pine Barrens (3.11), tandis que le critique, essayiste et réalisateur Peter Bogdanovich, qui joue également un savoureux personnage de psychanalyste «au carré » (il analyse l'analyste), se voit confier un épisode important de la saison 5. Dans la vingtaine de scénaristes ou de dialoguistes mis à contribution, on relève un groupe central semblable à celui des réalisateurs, avec quelques recoupements: Mitchell Burgess et Robin Green (celle-ci titulaire d'une licence de littérature anglaise de l'Université Brown et d'un master de creative writing de l'université de l'Iowa) ont écrit plus d'une vingtaine de scénarios. Matthew Weiner (12 scénarios dans les saisons 5 et 6) appartient au même milieu, de même que Terence Winter (plus de 20 scénarios en tout), ancien avocat né à Brooklyn, également titulaire d'une licence de lettres obtenue à NYU. Dans cette série conçue, écrite, produite et tournée sur les lieux même de l'action, le point de vue est majoritairement masculin. On compte deux femmes seulement parmi les scénaristes (Robin Green et Diane Frolov, qui travaillent la plupart du temps avec leurs compagnons respectifs, Mitchell Burgess et Andrew Schneider) et une seule réalisatrice, Lorraine Senna (Down Neck, 1.7).

4 Salués par la critique et plébiscitée par le public, Les Sopranos ont inspiré d'innombrables commentaires sur internet, une vingtaine d'ouvrages collectifs et de monographies (Gabbard ,2002; Lavery, 2006; Polan, 2009), au moins un colloque international organisé à l'Université Fordham en 2008 (Lavery, 2011), ainsi qu'un essai publié récemment en France par un critique de cinéma en vue (Burdeau, 2011). Les 
études disponibles traitent de sujets à la fois divers et circonscrits : la représentation de la mafia et de la communauté italo-américaine, l'articulation externe et interne avec les grands films de Coppola (la trilogie du Parrain,1972,1974 et 1990) ou de Scorsese (particulièrement Mean Streets, 1972, et Les Affranchis, 1990), la psychanalyse, les questions de genre, le machisme, le problème de la justice et de la morale, l'intertextualité et les enjeux esthétiques. Mais on constate surtout un consensus sur la qualité exceptionnelle de cette production. Emmanuel Burdeau, dans son essai inspiré et stimulant, rejoint le chœur des laudateurs en soulignant la dimension et la profondeur proprement cinématographiques d'une œuvre dont il salue l'ambition «monumentale " (Burdeau, 97). Seul Dana Polan semble adopter un point de vue plus circonspect, en décalage avec le consensus dominant : il constate le caractère inégal des 86 épisodes, et il évoque l'effet d'ironie et le "postmodernisme sardonique » d'une série qui se moque de tout et d'elle-même, et qui fuit comme la peste la morale commune et les leçons sérieuses (Polan, 132).

5 Tous s'accordent pour dire que le véritable sujet des Sopranos est l'Amérique d'aujourd'hui dans la mondialisation. Mais on pourrait aussi considérer que les Sopranos recyclent en les modernisant un ensemble de thématiques et de ressorts narratifs éprouvés par Hollywood depuis longtemps. Comme le notait jadis Robert Warshow dans deux articles célèbres, le gangster de cinéma incarne le "héros tragique » dans sa version américaine, puisque la société des États-Unis condamne les individus à la réussite matérielle et à l'angoisse qui accompagne cette course sans fin : "il est ce que nous voulons être et craignons de devenir» ("He is what we want to be and what we are afraid we might become ", Warshow, 100). Cette thématique étatsunienne, cette manière de chercher à penser ou à dire l'Amérique est validée par Chase lui-même dans le titre du dernier épisode Made in America : il s'agit bien d'une histoire américaine, du devenir d'une famille qui appartient à la classe moyenne supérieure, du moins par son mode de vie matériel. Cette famille est formée de Tony et Carmela Soprano et de leurs deux enfants Meadow et Anthony Jr. («A.J».). Tony, officiellement à la tête d'une entreprise de traitement de déchets ( waste management business ») est en réalité un responsable mafieux important du nord de l'État du New Jersey. C'est un gangster de fiction qui correspond en tous points à la typologie dressée par Warshow après la Seconde Guerre : il est sans éducation ni culture, et tout aussi impulsif et brutal dans ses loisirs que dans son « travail » (Warshow, 105-106). Tony souffre en outre de problèmes psychologiques, lesquels se manifestent par des crises d'angoisse et des malaises psychosomatiques. L'idée n'est pas vraiment originale, puisqu'au même moment une comédie hollywoodienne fait également des rapports entre la mafia et la psychanalyse son argument principal, avec Robert De Niro dans le rôle principal de Analyze This (Harold Ramis, 1999)². Ici encore, la variation psychanalytique ne fait que développer une thématique familière : le gangster de cinéma aussi était « solitaire et mélancolique », ce qui ne l'empêchait pas de donner une «impression d'expérience et de sagesse profonde " (" the impression of a profound wordly wisdom», Warshow, 106). Comme son prédécesseur hollywoodien, le gangster de télévision pourrait se réduire à une fonction essentielle : « faire du mal aux gens » (« he hurts people », Warshow, 101). 


\section{La culture des Sopranos}

6 Les références culturelles qui habillent, parcourent et structurent Les Sopranos suffisamment pour attirer l'attention se répartissent en trois ensembles. Le premier, le plus visible, c'est ce qu'on pourrait appeler la culture populaire -constituée ici d'un mélange de musique de variété ou de rock, d'airs d'opéra ou de chansons italiennes (la bande son ne comporte pas de musiques originales), de cinéma et de culture télévisuelle. Le second ensemble, c'est l'agrégat des éléments divers qui, par addition ou accumulation, forment le matériau qui alimente les conversations de tous les jours : nouvelles, faits divers plus ou moins spectaculaires, événements politiques nationaux et internationaux, aperçus sur les débats du moment. Enfin, il y a la culture scolaire ou universitaire, celle qui passe par les livres.

7 Les titres des 86 épisodes fournissent quelques indications sur cet ensemble hétéroclite et néanmoins cohérent. On remarque ainsi plusieurs allusions à la musique populaire ou au cinéma hollywoodien ${ }^{3}$, ainsi que quelques références à la littérature : Amour Fou (3.12) renvoie au récit d'André Breton (1937) et au film éponyme de Jacques Rivette (1968), Sentimental Education, l'épisode mis en scène par Peter Bogdanovich, à Flaubert (5.6) et Second Coming (6.9) à l'un des poèmes les plus connus de W.B. Yeats. Ce mélange est composé de chansons, de films, de personnages, de livres et d'éléments " canoniques ", de tout ce qui fait la culture commune de la génération d'après-guerre, celle qui a vécu l'explosion simultanée de l'enseignement supérieur et de la culture populaire. Comme Martin Scorsese dans Casino (1995), où le succès planétaire des Animals ("House of the Rising Sun», 1964) accompagne un dénouement sanglant livré dans le montage rapide, presque échevelé, de courtes scènes séparées, les producteurs des Sopranos utilisent le rock comme fond sonore du crime : l'épisode Kaisha (6.12), l'un des plus sombres, commence et s'achève sur les accents de "Moonlight Mile ", morceau énigmatique des Rolling Stones (Sticky Fingers, 1971). Le rock n'est pas incompatible avec mécanique implacable de la tragédie et du meurtre.

8 Certains commentateurs affirment que la série est l'une des plus « littéraires » jamais produites, en raison du grand nombre d'allusions aux classiques de la littérature américaine et mondiale dans la trame narrative et dans les dialogues. David Lavery, dans son étude des saisons 4 et 5 , relève au moins une vingtaine de mentions des grandes œuvres littéraires du passé. Flaubert est évoqué au moment où Carmela trompe son mari avec un responsable du lycée que fréquente son fils (Sentimental Education, 5. 6). Meadow et A.J. s'escriment avec les œuvres que leurs professeurs leur demandent de lire : l'Étranger pour Meadow, Lord of the Flies ou Animal Farm pour A.J. Ailleurs, on entend Janice, la sœur caractérielle de Tony, s'exclamer soudainement, et hors de propos: "Ah, Bartleby, Ah, humanity »... - en réaction à la dépression dont souffre une veuve inconsolable (No Show, 4.2). Ces allusions sont distribuées d'une manière presque régulière dans l'ensemble des six saisons. Lorsque Tony se retrouve à l'hôpital à la suite d'une blessure qui a failli lui coûter la vie, sa fille Meadow lui récite la prière irrévérencieuse de Jacques Prévert : "Notre Père qui êtes aux cieux, restezy... » (Join the Club, 6.2). Dans l'un des épisodes les plus marquants de la première saison, Tony emmène Meadow pour une tournée des universités de Nouvelle-Angleterre en vue d'une éventuelle inscription l'année suivante. À Bowdoin College, dans le Maine, le père et la fille sont accueillis par une phrase de Hawthorne, ancien élève de l'institution: "No man can wear a face to himself and another to the multitude, without finally getting 
bewildered as to which one is true " (College, 1.5). Quelle est la fonction de cette mise en exergue visuelle d'un passage de la Lettre écarlate (chapitre XX), où Hawthorne évoque le «mal subtil » qui ronge Dimmesdale? S'agit-il, comme dans Scarface, Shame of the Nation (Howard Hawks, 1931), Public Enemy (William Wellmann, 1931) ou Little Caesar (Mervyn Leroy, 1931), d'administrer une leçon de morale préventive, en l'occurrence que les malaises psychosomatiques de Tony sont la punition qu'il mérite pour sa duplicité ? Ou de conforter l'ambiance, voire les aspirations littéraires de la série? Comme nous allons essayer de le montrer, on trouve dans Les Sopranos un fil conducteur, discret mais solide et quasi permanent, qui nous ramène sans cesse aux problèmes sommairement indiqués plus haut et qui esquisse un ensemble de prises de positions assez original dans le monde des séries télévisées.

\section{Littérature et éducation}

9 La question de la transmission de la littérature par le système éducatif est un leitmotiv: dès que l'on évoque une œuvre, l'école ou l'université ne sont jamais loin, et inversement. C'est que l'histoire de Tony Soprano est celle de l'accès impossible, ou à tout le moins empêché, au savoir et à la culture. Lui-même n'a pas fréquenté l'université, mais cela ne l'empêche pas d'énoncer les principes d'un père de famille responsable : «Si tu veux aller à l'université », prévient-il son fils A.J., " va falloir que tu te farcisses des bouquins» ("If you wanna go to college, you gotta crack the books») (Full Leather Jacket, 2.8)... Le fils de Tony, adolescent attachant mais caricatural par son égocentrisme et son indécision chronique, connaît une scolarité difficile, ce qui fournit aux producteurs maintes occasions de commentaires sur la littérature et son enseignement: œuvres à lire, essais à rédiger, la littérature est manifestement au centre du processus éducatif. Mais si Meadow, la sœur aînée, réussit à se faire admettre dans la prestigieuse Université Columbia, il n'en va pas de même pour A.J., adolescent indifférent, voire réfractaire à la culture et au prestige social auquel elle est censée donner accès.

Le rapport familial à la littérature se joue à l'intérieur d'une conception plutôt traditionnelle. À l'occasion d'un dîner qui réunit Meadow, son frère A.J., ses parents et quelques camarades de Columbia, le spectateur assiste à un débat de plus en plus animé, et qui est de nature littéraire (Eloise, 4.12). A.J. n'a obtenu qu'une note médiocre pour un devoir sur la nouvelle de Melville Billy Budd, parce qu'il n'avait pas vu l'aspect homo-érotique du récit. Carmela, en bonne mère de famille, est piquée au vif par cette injustice flagrante : elle en a assez de toutes « ces bêtises gay » (« this gay nonsense ») qui " imprègnent le système éducatif et la télévision », («this stuff pervades our educational system and tv...»). Billy Budd, poursuit-elle, n'est rien d'autre que l'histoire d'un « jeune homme innocent persécuté par un chef méchant " («an innocent young man persecuted by a mean boss »). D'ailleurs, précise-t-elle, elle a vu le film avec Terence Stamp. Mais elle ignore que celui-ci a aussi joué des personnages moins innocents que le héros de Melville: comme le fait observer l'un des camarades de Meadow, l'acteur anglais incarne un transsexuel dans Priscilla reine du désert (Stephan Elliott 1994). Et tandis que Tony essaie de mettre les rieurs de son côté, en glissant que Billy Budd était certainement «le fleuriste du bateau», Meadow, bonne élève, rappelle que l'interprétation homosexuelle est connue depuis les années soixante par les analyses de Leslie Fiedler. Carmela, pensant que celui-ci est une femme, rejette l'objection, car une 
femme ne peut selon elle rien comprendre à ce genre de situation. Cette irruption humoristique des queer studies dans le monde des gangsters est un exemple de la roublardise des producteurs. Ceux-ci hésitent en effet rarement à se jouer des stéréotypes politiquement et socialement sensibles, qu'il s'agisse du sujet apparemment central dans la série (la communauté italo-américaine et ses rapports avec la «mafia ») ou de sujets connexes (la télévision, la famille, le sexe, le cinéma, la psychanalyse, les noirs et les minorités...), ce qui leur permet de titiller les susceptibilités libérales ou politiquement correctes d'une partie de leur public.

11 Les positions respectives de Tony et de Carmela à propos de l'éducation littéraire de leurs enfants - distance goguenarde pour le premier, prudence et bon sens maternel pour la seconde, nous renseignent sur les rapports qu'entretiennent les deux personnages principaux avec la culture. Tony ne lit pas de livres - sauf l'ouvrage illustré sur les dinosaures que sa femme lui apporte à l'hôpital, mais il regarde beaucoup et attentivement la télévision, car c'est elle qui lui procure les nourritures psychiques dont il a besoin. Il est captivé par les westerns classiques ou les émissions du History Channel, mais il sait qu'il ne parviendra jamais à combler ses lacunes et il attend de ses enfants qu'ils le fassent à sa place. Malgré tout, Tony entretient avec ses deux genres de prédilection un rapport proprement « littéraire ", puisqu'il y puise des leçons de vie, ou à tout le moins la confirmation de certaines valeurs qui pourraient être les siennes : Gary Cooper dans High Noon est l'incarnation parfaite du « strong silent type » auquel il aspire à ressembler (c'est d'ailleurs le titre du dixième épisode de la saison 4), de même que Winston Churchill, dont la force, peut-être moins silencieuse, est moralement égale à celle du shérif qui ne connaît pas la peur. La réalité historique se confond ainsi avec la fiction, Churchill égale Gary Cooper : deux images en noir et blanc d'une époque évidemment révolue, des hommes comme on n'en fait plus. Les producteurs procèdent ainsi à une mise en abyme intéressante, où l'on voit le gangster fictif (Tony) redevenir l'enfant qui se rejoue le film en se concentrant sur la seule chose qui compte: la figure que fait celui qui tire ou qui tombe. Comme l'observait déjà Warshow, «le héros est celui qui a l'air d'un héros" («A hero is one who looks like a hero ", Warshow, 123).

12 Contrairement à son époux, Carmela s'intéresse aux livres. Dans la deuxième saison, par exemple, on la voit absorbée par la lecture de Memoirs of a Geisha, bestseller d'Arthur Golden (1997) qui la met peut-être sur la voie d'une meilleure compréhension de sa propre position d'épouse entretenue et dépendante. Plus tard, au moment où elle se sépare de Tony, Carmela se laisse séduire sans trop de résistance par Wegler, le directeur des études du lycée privé que fréquente son fils. Wegler l'impressionne par sa culture. Il lui fait découvrir Mme Bovary, roman qu'elle a du mal à lire mais qui l'attire confusément, ne serait-ce que parce que son amant lui offre l'édition princeps de la Modern Library. Carmela sent que le cadeau de Wegler « fera bien » dans son intérieur, lequel a été soigneusement conçu par la production comme la projection matérielle des aspirations sociales attribuées au personnage (Martin, 29). Pour reprendre une formule de H.L. Mencken vitupérant le matérialisme des années 1920, Carmela ne dépasse guère le "stade décoration intérieure de la culture" ("the interior decorator stage of culture ", Mencken, 178). En même temps, et comme son mari, Carmela ressent un manque spirituel. Ceci ne l'empêche pas, à l'occasion, de connaître l'expérience esthétique : lors d'une visite au Metropolitan Museum of Art de New York avec sa fille, elle fond en larmes devant le Mariage mystique de Ste Catherine de Jose de Ribera (1648) ( Amour Fou, 3.12). Plus tard, lors de son voyage à Paris, elle n'est pas loin de la transe : 
le regard sans cesse tourné vers le haut, elle semble terrorisée à l'idée de laisser échapper la moindre miette du spectacle (Cold Stones, 6.11). Mais lorsque Carmela est confrontée au Beau authentique, le ridicule n'est jamais loin. Les auteurs se montrent décidément sans pitié pour leur personnage : si le beau lui est accessible sans concept, c'est surtout parce qu'elle n'y comprend rien.

On pourrait affirmer que Tony et Carmela partagent les mêmes certitudes instinctives : l'apprentissage de la littérature - modèle de la culture en général - demande des efforts (qu'eux-mêmes n'ont pas consentis) et permet l'accès aux sphères supérieures, aux professions respectables, celles qui assurent des revenus confortables par des moyens honnêtes et sans risques. La culture ainsi acquise ouvre la voie qui mène au prestige social mais aussi à d'autres dimensions de la vie : l'expérience du beau pour Carmela, les hauteurs du courage pour Tony. En même temps, ils sont en proie au doute : l'un comme l'autre, ils sentent que ni les satisfactions matérielles, ni le succès universitaire de leur fille ne sont susceptibles de satisfaire leurs exigences profondes. Ils constatent également que les bénéfices symboliques et éthiques qu'ils associent à la détention d'un capital culturel acquis sont presque systématiquement démentis par l'expérience. L'amant de Carmela a beau laisser traîner un exemplaire des lettres d'Héloïse et Abélard dans sa salle de bains, il se révèle vite tel qu'il est: un homme médiocre. Lorsque le docteur Cusamano, médecin de famille et voisin des Sopranos, invite Tony à se joindre à une partie de golf au country club, Tony accepte d'autant plus volontiers qu'il espère devenir membre du club en question et peut-être se fondre dans la classe supérieure dont il envie l'aisance sociale, les capacités intellectuelles et la culture. Mais il devra vite déchanter, s'apercevant qu'il n'a été invité que pour satisfaire la curiosité malsaine et condescendante d'un petit groupe de médecins, d'avocats et de banquiers ordinaires pour le voyou qu'il est. Son rêve d'ascension sociale et culturelle se transforme en humiliation: il vient d'être traité comme un ours de foire (" a fucking dancing bear ») déclare-t-il, furieux, peu après l'incident (A Hit Is a Hit, 1.10). Cusamano, hypocrite et mesquin jusqu'au bout, ne tarde pas trop à lui faire savoir qu'il est sincèrement désolé, mais que le country club ne prend plus d'inscriptions.

\section{Prestige social, supériorité morale et maîtrise langagière}

14 L'instance intellectuelle ou cultivée trouve plusieurs incarnations dans Les Sopranos. On peut citer Father Phil, prêtre catholique revêtu des oripeaux du savoir et de la religion, mais il est faible face aux tentations de la table - auxquelles il succombe -, et à celles du lit - auxquelles il résiste parce qu'elles le terrorisent (College, 1.5). Les intellectuels des Sopranos sont la plupart du temps moqués et traités avec la même dérision que les gangsters. Le cercle des amis et confrères qui entourent Jennifer Melfi, par exemple, les hommes qu'elle fréquente, comme son ex-mari ou son psychanalyste, n'ont rien d'exceptionnel en tant qu'êtres humains : ils sont tout aussi désemparés que le commun des mortels, souvent prétentieux et dénués de courage. Dans la deuxième saison, le témoin fortuit d'un assassinat particulièrement sauvage commis par Tony lui-même se rend spontanément à la police pour apporter sa contribution de citoyen au respect de la loi et de l'ordre. C'est un universitaire, que l'on découvre un peu plus tard chez lui, plongé dans la lecture de l'ouvrage de Robert Nozick Anarchy, State and Utopia (1974), bible de l'ultralibéralisme "minarchiste ». Dès qu'il apprend que l'affaire est liée au 
clan des Sopranos, il se rétracte piteusement (Bust Out, 2.10). À un autre moment, Chris Moltisanti, neveu et successeur putatif de Tony, qui s'est mis en tête de devenir écrivain, agresse physiquement son professeur d'écriture. Celui-ci, le visage en sang, se tourne vers ses élèves en hurlant: «Des écrivains tous autant que vous êtes, et vous n'avez pas levé le petit doigt! » ("An entire room full of writers and you did nothing!» Mayham, 6.3). Dans le contexte de la série, ce message à répétition est clair: les gangsters sont peut-être des brutes incapables de maîtriser leurs pulsions, mais les intellectuels sont des lâches.

Il y a une exception: Jennifer Melfi. D'une certaine manière, c'est elle qui incarne l'autorité culturelle supérieure du début à la fin de l'histoire. C'est elle qui cite Yeats ( " the center cannot hold... ») et qui corrige les erreurs de Tony lorsque celui-ci mutile les expressions les plus courantes: "revenge is like serving cold cuts» croit-il pouvoir affirmer (Cold Cuts, 5.10), malmenant la métaphore idiomatique qu'il n'a pas correctement enregistrée ("revenge is a dish best served cold»). Jennifer Melfi tient un discours pédagogique parfois pompeux, ce qui a pour effet d'exaspérer son patient et de faire sourire le spectateur, mais elle connaît aussi le doute et l'hésitation. Parmi les personnages principaux, elle est pourtant la seule à atteindre une certaine noblesse. Cela se passe dans l'un des épisodes les plus "sérieux ", Employee of the Month (3.4). Femme libre et indépendante, exerçant une profession reconnue - le contraire de Carmela -, elle est victime d'un viol dans le parking de son cabinet. Le violeur est arrêté par la police, mais rapidement relâché en raison d'une erreur de procédure. Tentée de demander vengeance à Tony Soprano, Jennifer Melfi résiste et préfère souffrir en silence et dans la légalité. Cette décision rationnelle et morale confère au personnage une grandeur dont il n'y a pas d'autre exemple dans Les Sopranos. Ainsi se dessine une sorte d'équivalence concrète entre la culture apprise et la hauteur morale, une preuve par le comportement, qui ne dure que quelques secondes, dans un échange intime et presque silencieux entre l'analyste et son patient. Tony Soprano se doute de quelque chose mais il ne saura rien et son offre de protection restera virtuelle, tandis que Jennifer Melfi s'en tient au pacte social et féministe: pas de vengeance privée, pas de compromission avec un criminel, pas d'acte de soumission au mâle (Roche).

Pour Les Sopranos, l'acquisition de la culture, qui passe par la lecture, notamment celle des grandes œuvres, est donc présentée comme l'instrument d'émancipation principal. C'est elle qui permet l'accès à l'enseignement supérieur, aux "arts libéraux", aux professions dignes, à celles dont tous les parents rêvent pour leurs enfants, même si la supériorité morale à laquelle on pourrait s'attendre n'accompagne pas nécessairement l'élévation du niveau culturel. Cette constatation sociologique prend la forme d'une célébration de la littérature et de la culture, elle-même inscrite dans un pluralisme non discriminatoire qui est celui des producteurs de la série et de leur génération. Mais elle prend aussi un tour spécifique qui se manifeste à de nombreuses reprises. Parmi les incidents littéraires, on cite souvent le dialogue entre Tony et Bobby, son futur beaufrère, gangster obèse et naïf, qui confond Quasimodo et Nostradamus. Pour une fois, c'est Tony qui joue les correcteurs : "Nostradamus and Notre-Dame is two different things complete» (For All Debts Publics and Private, 4.1). Cette rectification d'une référence culturelle erronée, elle-même livrée dans un anglais peu châtié, est un exemple parmi d'autres, mais elle nous met sur la voie: Les Sopranos, c'est aussi une affaire de langue.

Comme l'atteste la prolifération bouffonne de l'adverbe "fucking" dans un usage explétif, ou celle du substantif polyvalent "shit», ces gangsters qui tuent presque 
comme ils respirent se contentent d'une expression primitive et fautive. Les impropriétés (malapropisms) et les lapsuscomiques constituent d'ailleurs l'une des marques de fabrique des Sopranos. On a vu que Chris Moltisanti, le neveu de Tony, avatar masculin du bovarysme incarné par Carmela, se pique d'écrire. Mais lorsque l'écrivain en herbe veut commenter l'action et dire " diviser pour régner ", il confond deux mots savants : ce que veulent les parrains, affirme-t-il doctement, c'est créer un peu de " dysenterie dans les troupes » (" dysentry among the ranks ») (The Weight, 4.4).

Certes, la multiplication des jurons permet certaines trouvailles. Ainsi la définition du golf par Furio, éphémère « soldat » italien dont Carmela s'amourache platoniquement, traduit-elle efficacement le scepticisme que suscite parfois ce sport: la formule "stupida fucking game " (He Is Risen, 3.8), a connu une indéniable fortune. Il n'empêche : la langue précise et nuancée reste l'apanage des bourgeois, de ceux qui ont eu la chance de faire des études. Nous avons vu que Tony lui-même répète servilement certaines expressions entendues de la bouche du Dr. Melfi lors des séances d'analyse, souvent en les travestissant d'une manière grotesque. Quand il ne comprend pas une proposition formulée en termes trop abstraits, il exige une traduction : «English? " (Full Leather Jacket , 2.8). Tony affirme ainsi sa soumission à une autorité qui l'impressionne d'autant plus qu'elle lui est inaccessible, celle que confère l'aisance syntaxique et lexicale.

Le jeu sur les niveaux de langue prend un tour insistant à l'occasion d'une scène intime, dans l'un des épisodes mis en scène par Steve Buscemi (Pine Barrens, 3.11). Meadow, à peine remise d'une rupture douloureuse avec son petit ami précédent, un jeune intellectuel antipathique, est tombée amoureuse de son ami d'enfance, Jackie Jr., le fils du parrain décédé qui avait ouvert la voie à l'ascension rapide de Tony Soprano. Jackie Jr. est beau, attentionné, délicat et il affirme sa volonté de faire des études. Meadow, devenue étudiante à Columbia, est grippée ce jour-là et décide de garder la chambre. $\mathrm{Ne}$ pouvant sortir, les deux amants tuent le temps en jouant au scrabble, et l'on comprend que Jackie Jr. s'ennuie. Dans un gros plan appuyé, le spectateur partage le regard de Meadow sur les mots de Jackie Jr. - "poo ", «ass», «the »... Ce jeune homme au vocabulaire affligeant et à la psychologie régressive révèlera bientôt sa véritable nature, celle d'un paresseux violent, menteur et stupide. Il en mourra d'ailleurs quelques épisodes plus loin, éliminé en raison de son comportement imprévisible et dangereux pour le groupe. Meadow, étudiante studieuse, est entretemps devenue l'incarnation secondaire de la légitimité culturelle, après le Dr. Melfi : elle n'a pas encore la maîtrise de celle-ci, mais elle y aspire manifestement, s'exprimant dans une langue qui contraste de plus en plus avec celle de sa famille. Inversement, les conséquences de la paresse et de l'ignorance sont plusieurs fois soulignées. Ainsi, lorsque Chris Moltisanti prend des leçons de théâtre, il se révèle incapable d'apprendre par cœur un texte de Tennessee Williams tiré de la Ménagerie de verre. En remplacement, il impose au professeur une scène mélodramatique de La Fureur de vivre, le film de Nicholas Ray, qu'il connaît par cœur. Chris préfère se prendre pour James Dean jusqu'à perdre la maîtrise de ses émotions plutôt que d'incarner un personnage qui ne lui dit rien: Chris ne veut pas et ne peut pas partir d'un texte imprimé (Big Boys Don't Cry, 2.5).

20 C'est peut-être cette insistance madrée sur la maîtrise de la langue, acquise par la fréquentation des œuvres et la lecture qui fait l'originalité des Sopranos. Certes, la question de la langue des criminels a toujours constitué un ressort important dans les films de gangsters depuis l'avènement du cinéma parlant. Si la langue stylisée des 
premiers gangsters de cinéma nous semble conventionnelle aujourd'hui, elle avait malgré tout une fonction simple: faire sentir aux spectateurs le contraste édifiant entre l'éducation (le bien) et l'ignorance (le mal) - thème central de la leçon de morale qui accompagnait les classiques au moment de leur sortie au début des années 1930. Tony connaitt ces films, et surtout l'un d'entre eux, Public Enemy, cité plusieurs fois. Dans Les Sopranos, cependant, et moyennant l'addition d'éléments sociologiques et psychanalytiques, la maîtrise de soi n'est pas une affaire de morale, de choix conscient et responsable, de séparation nette entre le bien et le mal - comme dans les films fondateurs du genre -, mais elle commence par la maîtrise de la langue, que ne possède aucun des gangsters de la série. C'est en cela que le dialogue constitue une originalité thématique et stylistique : libéré des contraintes de l'autocensure préventive à laquelle les premiers films de gangsters étaient soumis bien avant la mise en place de la Production Code Administration en $1934^{4}$, mais aussi prenant ses distances avec les modèles établis par Coppola ou Scorsese, l'œuvre télévisuelle imaginée et produite par David Chase sonne parfois comme un rappel de l'importance de l'enseignement et de la pratique de la langue grâce à la fréquentation de la littérature. Tous ces gangsters de télévision cherchent sans cesse à comprendre, à s'exprimer, à mettre en ordre l'expérience et le réel, alors même qu'ils sont enfermés dans leur ignorance et leur maladresse langagière. Les personnages se meuvent dans la pénombre d'une parole approximative, qui les prive des moyens d'expression, de compréhension et d'analyse dont ils ont besoin : c'est l'aspect performatif des Sopranos, une série qui fait ce qu'elle entend démontrer.

21 D'un côté, on est proche du genre parodique si populaire en France depuis le début de la Ve République grâce à des films comme Le Cave se rebiffe (Gilles Grangier, 1961)ou Les Tontons flingueurs (Georges Lautner, 1963). Les personnages de Paulie Walnuts (Tony Sirico) ou de Silvio Dante (Steven Van Zandt) sont, mutatis mutandis, les équivalents des frères Volfoni (Bernard Blier et Jean Lefebvre) et de $\mathrm{M}^{\mathrm{e}}$ Folace (Francis Blanche), de même que Tony Soprano (James Gandolfini) ressemble parfois à M'sieur Fernand (Lino Ventura). Mais l'autorité à la fois savante et gouailleuse des gangsters de convention imaginés par Michel Audiard a en réalité peu en commun avec l'expression hésitante et brouillonne des gangsters « réalistes » de David Chase. Tandis que les premiers sont les porte-voix d'un auteur féru de culture classique et d'argot parisien, les seconds passent le plus clair de leur temps à balbutier dans le noir, même si les dialoguistes des Sopranos, comme Michel Audiard, ne se privent pas d'exploiter ces travers à des fins chansonnières. Mais dans Les Sopranos, œuvre vouée à l'ironie et à la dérision, il subsiste une part de sérieux: celle-ci se joue autour des capacités langagières que donne la maîtrise d'un capital culturel acquis par l'étude et grâce à l'école et à l'université, et qui est celle que partagent l'ensemble des producteurs, réalisateurs, scénaristes et dialoguistes de la série.

22 Si nous saisissons un peu mieux le sens possible de l'atmosphère littéraire entretenue par les producteurs des Sopranos, il nous reste à nous demander dans quelle mesure cette insistance sur la littérature et sur la culture légitime appelle ou induit une prise de position sur la nature de la culture populaire, notamment sous sa forme audiovisuelle. 


\section{Culture légitime et culture audiovisuelle}

23 Carmela est attirée par la littérature, l'art, la beauté. Chris Moltisanti se considère comme écrivain et scénariste, alors que Tony, qui se maintient à une distance respectueuse, ne fréquente la littérature que par l'intermédiaire de ses enfants et se contente des westerns et des émissions du History Channel. Et tandis que Meadow fraye son chemin dans la culture scolaire, A.J. s'y perd... La pratique culturelle dominante de cette société de gangsters reste la télévision et la vidéo, ce qui se manifeste par l'installation d'un équipement luxueux de home cinéma au cœur du grand salon de la résidence familiale ainsi que par l'omniprésence des écrans dans la plupart des intérieurs. Dans la famille, on constate une palette de comportements variés: l'aspiration au beau (Carmela), la recherche du modèle héroïque (Tony), l'application émancipatrice (Meadow), le refus adolescent (A.J.) et la prétention artistique (Chris). Pourtant, entre la régression infantile (Tony qui se rejoue le film du héros et aime les dinosaures, Chris l'écrivain présomptif incapable de maitriser ses pulsions et A.J enfermé dans son conformisme adolescent), l'émotion spontanée (Carmela) et l'apprentissage appliqué (Meadow), le nombre des options est en réalité limité. Ainsi, le problème posé, d'une manière assez classique, est celui des vertus de l'éducation « libérale » qui émancipe par rapport aux effets non maitrisés de la culture de masse qui aliène et réprime. C'est peut-être la manière qu'ont trouvée Les Sopranos pour s'interroger sur leur propre statut en tant que production culturelle.

Observons pour commencer que bien peu nombreux sont ceux qui se risqueraient aujourd'hui à formuler ouvertement ou sérieusement des réserves sur la valeur esthétique de la culture populaire. C'est un fait entendu que les distinctions entre « haute culture » et " culture populaire " n'ont plus guère de sens, et l'on hésiterait à parler aujourd'hui d'un "fossé infranchissable " ( great divide ») entre la culture de masse et l'art véritable (Howe, 503), entre le kitsch, «mot allemand pour désigner la culture de masse " (" ...a German term for mass culture ", Macdonald, 59), et la haute culture. Reconnaissons qu'il est en effet tentant de voir dans Les Sopranos une sorte d'équivalent de la littérature, un grand roman contemporain, répondant au même besoin de récit, avec le même souci de complexité, de cohérence organique et d'ouverture. Certains considèrent même Les Sopranos comme l'avènement inattendu et « postmoderne » du " Grand Roman Américain » que tout le monde espère depuis 1945 (Nelson, 52-53).

On peut en effet reconnaître à cette série les qualités d'une œuvre narrative ambitieuse et réussie. Par sa dimension, son élan, et surtout par son traitement détaché et nuancé des bribes de réalité qui la nourrissent, elle correspond largement au projet romanesque tel que le définissait le dernier Barthes: cette transformation des notations, du temps qu'il fait, des ambiances « New Jersey ", de la «nuance » en trame romanesque, avec sa part d'irrésolu, de vide, d'inconnu, de vertige (Barthes, 2003). Mais la véritable force des Sopranos se situe peut-être ailleurs, dans le traitement parfois approfondi et courageux de certains thèmes tirés de l'actualité : l'homophobie est l'un d'entre eux, sans doute le plus surprenant. Lancée dès la fin de la saison 5 (Unidentified Black Males, 5.9, épisode diffusé au printemps de l'année 2004), la thématique se développe dans la saison suivante, et on ne peut s'empêcher de remarquer la coïncidence avec l'apparition du mariage gay au premier plan des problèmes évoqués dans le débat politique national ${ }^{5}$. La trame est la suivante: Vito Spafatore, l'un 
des capitaines de l'équipe de Tony Soprano est un gangster normal, c'est-à-dire brutal, cupide et paresseux, mais contre toute attente et contre les stéréotypes (car il est corpulent, marié et père de famille), il est aussi homosexuel. Découvert par hasard, Vito s'enfuit dans le New Hampshire, où il vit une brève idylle avec un restaurateur qui est par ailleurs pompier bénévole (Johnny Cakes, 6.8 et Moe'n Joe, 6.10). Mais Vito s'ennuie à la campagne et, incapable de travailler, décide finalement de rentrer en ville. Il sera puni et assassiné par les «siens » dans l'une des scènes les plus insoutenables de la série (Cold Stones, 6.11). Voici donc Les Sopranos qui prennent position et qui condamnent l'homophobie : les conséquences possibles du rapprochement entre les conservateurs évangéliques, farouchement opposés au mariage homosexuel, et certains milieux catholiques, qui ne le sont pas moins, se trouvent ainsi mises en drame d'une manière spectaculaire, puisque ce sont les gangsters italiens, incarnation de la «culture » catholique la plus primitive, qui servent à la fois de panneau indicateur et de repoussoir (De Stefano, 122).

Sur le plan formel, la série témoigne d'une richesse et d'une diversité indéniables. Mais si l'on excepte le générique, les illustrations musicales, les trois ou quatre intérieurs récurrents (le bar de strip-tease Bada Bing, l'arrière-boutique de la charcuterie Satriales', la villa de Tony Soprano), peut-on pour autant parler d'un style cinématographique ou télévisuel propre? Ajoutons que Les Sopranos fonctionnent en pleine conformité avec les lois du genre de la série télévisée: plusieurs intrigues parallèles par épisode, personnages fixes à qui il arrive des choses, mais qui n'évoluent pas autrement que par les circonstances ou les accidents, régularité des thèmes. Certes, on constate la présence de certains éléments perturbateurs: c'est ainsi que le spectateur ne saura jamais ce que devient le gangster russe qui échappe à son exécution dans l'épisode Pine Barrens (3.11). Administrées à petites doses, ces coquetteries narratives n'enlèvent rien au fonctionnement général ni à l'effet de la série sur les spectateurs, à savoir l'installation rapide d'un état de dépendance. Que va$\mathrm{t}$-il arriver à Tony? Va-t-il être puni pour ses crimes? Carmela va-t-elle finir par tromper son mari? Le Dr Melfi succombera-t-elle à son patient? On observe que les enjeux stratégiques, les jeux de pouvoir au sein de la mafia, les luttes de territoire et d'influence, tout ce déjà-vu de la mythologie fabriquée depuis les années 1920 autour de la criminalité organisée, semblent compter moins que les relations affectives ou les rapports de force entre les individus. La série mise d'une manière fondamentale et structurelle sur des ressorts psychologiques traditionnels et éprouvés, comme naguère les feuilletons radiophoniques et autrefois des romans populaires publiés par épisodes dans les journaux, les magazines ou les almanachs.

On pourrait donc dire que Les Sopranos évoquent en passant la question de leur propre valeur artistique par comparaison avec la littérature, mais sans trop y croire ni s'y attarder. Si la série intègre la culture légitime, dans sa version scolaire, si elle reconnaît la valeur de la culture littéraire et le prix des efforts nécessaires pour l'acquérir, si elle paraît particulièrement attentive à la question linguistique, aux enjeux des niveaux de langue, elle reste une série télévisée. Certes, elle a l'audace d'aller le plus loin possible dans la noirceur et la violence, mais elle ne cherche que rarement à subvertir les conventions génériques: il lui faut coûte que coûte s'attacher les téléspectateurs semaine après semaine, épisode après épisode. Les Sopranos ont sans doute, après d'autres tentatives, contribué à faire sauter davantage encore le verrou de la moralité narrative (le meurtre n'est pas puni, le mal triomphe), et ils engendreront vraisemblablement des séries plus sombres encore, et en même temps, ils ont du mal à 
dépasser le stade de la dérision: Jennifer Melfi connaît un moment de grandeur et d'héroïsme, mais elle n'échappe pas entièrement au ridicule. La série commente parfois l'actualité, mais c'est plus pour en tenir compte du point de vue factuel ou visuel (les tours jumelles disparaissent du générique après le 11 septembre 2001) que pour en tirer une matière romanesque. Cela ne l'empêche pas, parfois, de prendre position sur certains sujets brûlants, un peu à la manière d'un roman à thèse, en offrant « une version extrême d'un phénomène général » (Suleiman, 10) : c'est ce qui se passe, nous l'avons vu, avec la dénonciation de l'homophobie développée dans les deux dernières saisons. Il reste que, dans l'ensemble, elle ne dépasse pas les limites esthétiques et les contraintes du genre auquel elle appartient.

\section{Conclusion}

Dans l'avant-dernier épisode, Phil Leotardo, le nouveau boss de la famille new-yorkaise, se livre une fois encore à l'exercice préféré des gangsters : l'analyse de la conjoncture, la construction de l'avenir, l'élaboration d'une stratégie. La réunion se tient dans le café habituel, autour d'une table, et Phil Leotardo, pour clarifier la situation, préconise l'élimination des membres principaux du clan des Sopranos (The Blue Comet, 6.20). Son intervention est martiale pour le ton et le contenu mais fragile du point de vue de la langue : «Lemme tell you a couple of three things... ». Cette tentative de mise en ordre sera presqu'aussitôt démentie par les faits, car c'est lui-même qui sera exécuté dans une station essence, devant sa femme et ses petits-enfants. À la fin du dernier épisode, la famille Soprano se retrouve dans un restaurant. Tandis que Tony, Carmela et A.J. prennent connaissance du menu, le spectateur est témoin d'allées et venues vaguement inquiétantes autour d'eux. Au moment précis où Meadow, qui vient de garer sa voiture, s'apprête à entrer, l'écran devient noir ("cut to black») pendant quelques longues secondes avant que ne défile le générique, en silence : c'est la fin d'une série qui a dit ce qu'elle avait à dire sur le monde des gangsters et qui ne conclut pas, comme si les ressources étaient épuisées et qu'il n'y avait plus rien à ajouter. Comme nous avons essayé de le montrer, Les Sopranos nous laissent un plaidoyer objectif en faveur de la littérature et de son rôle, que la série présente comme irremplaçable pour l'acquisition de la maîtrise de la langue, de soi et des choses - même si, derrière le masque ricanant de la dérision, on sent pointer une nostalgie pour un monde qui est derrière nous.

\section{BIBLIOGRAPHIE}

BARTHES, Roland, La Préparation du roman I et II. Cours et séminaires au Collège de France (1978-1979 et 1979-1980), Paris, Le Seuil/IMEC, 2003. Séances du 2, 9 et 16 décembre1978, puis du 6, 13, 20 et 27 janvier 1980.

BURDEAU, Emmanuel, La Passion de Tony Soprano, Nantes, Editions Capricci, 2011.

DE STEFANO, George, " A 'Finook' in the Crew : Vito Spafatore, The Sopranos and the Queering of the Mafia Genre », dans TESR, 114-23. 
GABBARD, Glen O., The Psychology of the Sopranos : Love, Death and Betrayal in America's Favorite Gangster Family, New York, Basic Books, 2002.

HOWE, Irving, "Notes on Mass Culture", dans Bernard ROSENBERG et David MANNING WHITE, dir., Mass Culture : The Popular Arts in America, New York, The Free Press, 1957, 496-503 [Politics, printemps 1948].

LAVERY, David, « The Allusions of Television », 26 janvier 2006, http://flowtv.org/2006/01.

LAVERY, David, Douglas HOWARD et Paul LEVINSON, dir. The Essential Sopranos Reader, Lexington, KY, The University Press of Kentucky, 2011. [TESR ci-dessus et ci-dessous]

LAVERY, David, Reading the Sopranos : Hit TV from HBO, Londres et New York, IB Tauris Ltd, 2006.

MACDONALD, Dwight, “A Theory of Mass Culture”, dans Bernard ROSENBERG et David MANNING WHITE, dir., Mass Culture : The Popular Arts in America, New York : The Free Press, 1957, 59-73

[Diogenes, été 1953, 1-17].

MARTIN, Brett dir. The Sopranos : The Complete Book, New York, HBO/Time, 2007.

MENCKEN, Henry Louis, « American Culture », A Mencken Chrestomathy, New York, A. Knopf, 1949.

NELSON, Robin, « Author(iz)ing Chase », TESR, 41-53.

POLAN, Dana, The Sopranos, Durham, Duke University Press, 2009.

REDMOND, Thomas, «The Sopranos as Literature : The Art of Television », 9 mai 2008, sur le site associatedcontent.com, accès le 9 août 2011.

ROCHE, Nancy McGuire, « Honoring the Social Compact : The Last Temptation of Melfi », dans TESR. 104-113.

Rolling Stones (The), Sticky Fingers, 1971.

SULEIMAN, Susan, Le roman à thèse ou l'autorité fictive, Paris, PUF, 1981.

WARSHOW, Robert, The Immediate Experience: Movies, Comics, Theater and Other Aspects of Popular Culture, Boston, Harvard University Press, 2001. Édition originale : New York, Doubleday, 1962. [Ce recueil posthume contient les deux articles évoqués ici : "The Gangster as Tragic Hero », Partisan Review 2, 1948 et «The Gentleman With a Gun », Encounter, mars 1954, ce dernier sous le titre « Movie Chronicle : The Westerner »]

\section{NOTES}

1. Si nous empruntons à Pierre Bourdieu les expressions " culture légitime », « capital culturel » ou "distinction", devenues courantes depuis la publication d'ouvrages majeurs comme La Reproduction (1970) ou La Distinction (1979), nous n'entreprenons pas une analyse sociologique des Sopranos, œuvre de fiction née et déployée dans un environnement culturel et historique différent de celui qui avait occupé le sociologue français. Plus modestement, nous essayons de lire Les Sopranos à la lumière d'une problématique spécifique, et qui porte sur le discours que tient la série sur certains aspects de la vie sociale et culturelle des États-Unis au tournant du XXI siècle.

2. Le succès de ce film (assez médiocre) et celui de la série Les Sopranos incitent Warner Brothers à produire la suite trois ans plus tard Analyze That (Harold Ramis, 2002).

3. Dans la première catégorie, on relève notamment « Fortunate Son " (Creedence Clearwater Revival, 1969), «The Weight» (The Band,1968) ou « Remember When » (The Platters, 1959). Cette « signalétique » pop/rock est souvent superficielle, et parfois même sans rapport avec le contenu 
des épisodes en question. Dans la seconde catégorie, on trouve parmi d'autres From Where to Eternity ou Full Leather Jacket, allusions transparentes aux films de Fred Zinnemann (1953) et Stanley Kubrick (1987), respectivement.

4. La liste des Don'ts and Be Carefuls adoptée par la Motion Picture Association en 1927 en réaction aux multiples tentatives de censure des films, notamment au niveau des villes ou des États, constitua la base du Production Code adopté en 1930 et renforcé par la création d'un bureau spécifique en 1934 : les premiers films de gangsters parlants ne comportent pas d'argot grossier («profanity»).

5. Aux élections générales de novembre 2004, en réponse à des questions référendaires posées dans 11 États, les électeurs avaient rejeté le mariage homosexuel à des majorités supérieures à $60 \%$.

INDEX

Thèmes : Trans'Arts

\section{AUTEUR \\ BERNARD GENTON}

Université de Strasbourg 